\title{
On the sdOB primary of the post common-envelope binary AA Doradus (LB 3459) ${ }^{\star, \star \star}$
}

\author{
S. Klepp and T. Rauch
}

\begin{abstract}
Institute for Astronomy and Astrophysics, Kepler Center for Astro and Particle Physics, Eberhard Karls University, Sand 1, 72076 Tübingen, Germany e-mail: rauch@astro.uni-tuebingen.de
\end{abstract}

Received 14 March 2011 / Accepted 6 June 2011

\begin{abstract}
Context. AA Dor is an eclipsing, post common-envelope binary with an sdOB-type primary and a low-mass secondary. Eleven years ago, an NLTE spectral analysis showed a discrepancy in the surface gravity that was derived by radial-velocity and light-curve analysis, $\log g=5.21 \pm 0.1\left(\mathrm{~cm} / \mathrm{s}^{2}\right)$ and $\log g=5.53 \pm 0.03$, respectively.

Aims. We aim to determine both the effective temperature and surface gravity of AA Dor precisely from high-resolution, high-S/N observations taken during the occultation of the secondary.

Methods. We calculated an extended grid of metal-line blanketed, state-of-the-art, non-LTE model atmospheres in the parameter range of the primary of AA Dor. Synthetic spectra calculated from this grid were compared to optical observations.

Results. We verify $T_{\text {eff }}=42000 \pm 1000 \mathrm{~K}$ from our former analyses and determine a higher $\log g=5.46 \pm 0.05$. The main reason are new Stark-broadening tables that were used for calculating of the theoretical Balmer-line profiles.

Conclusions. Our result for the surface gravity agrees with the value from light-curve analysis within the error limits, thereby solving the so-called gravity problem in AA Dor.
\end{abstract}

Key words. stars: abundances - stars: atmospheres - binaries: eclipsing - stars: early-type - stars: individual: AA Dor (LB 3459) stars: fundamental parameters

\section{Introduction}

AA Dor is a close, eclipsing, post common-envelope binary system with an sdOB-type primary star and an unseen low-mass companion. The orbital period is 0.2615397363 (4) d (Kilkenny 2011 ) and the inclination is $i=89.21 \pm 0.30$ (Hilditch et al. 2003). A detailed introduction to the system and previous analyses is given in Rauch (2004), and we summarize results of previous spectral analyses of the primary in Table 1 .

Rauch (2000) encountered the problem of his spectroscopically determined surface gravity $\log g=5.21 \pm 0.1$ not matching $\log g=5.53 \pm 0.03$ determined from light-curve analysis (Hilditch et al. 1996). Hilditch et al. (2003) present an improved photometric model and derive $\log g=5.45-5.51$. The reason for the $\log g$ discrepancy is unknown. Fleig et al. (2008) find a slightly higher $\log g=5.3 \pm 0.1$ but the discrepancy remains. A recent analysis by Müller et al. (2010) has apparently solved the $\log g$ problem by finding $\log g=5.51 \pm 0.05$.

Müller et al. (2010) do not consider that the He I lines (as well as other lines of low-ionized species, e.g. of $\mathrm{Mg}$ II, Fig. 1) are too strong in the models at their favored parameters $T_{\text {eff }}=37800 \pm 500 \mathrm{~K}$ and $\log g=5.51 \pm 0.05$, as demonstrated in Fig. 1. Increasing the $\mathrm{He}$ abundance to better fit He II $\lambda 4686 \AA$ also results in a much stronger He $\mathrm{s} \lambda 4471 \AA$, which then disagrees with the observation. We mention that

* Based on observations made with ESO Telescopes at the Paranal Observatories under programme ID 66.D-1800.

$\star \star$ Tables 2 and 3 are available in electronic form at http: //www . aanda.org
Fleig et al. (2008) evaluate the ionization equilibria of $\mathrm{C}$ III/C IV, $\mathrm{N}_{\text {III }} / \mathrm{N}_{\text {IV }}, \mathrm{O}_{\text {III }} / \mathrm{O}_{\text {IV }}, \mathrm{P}_{\text {IV }} / \mathrm{P}_{\mathrm{V}}$, and $\mathrm{S}_{\text {IV }} / \mathrm{S}_{\mathrm{V}}$ in the $\mathrm{FUV}$ wavelength range and find $T_{\text {eff }}=42000 \pm 1000 \mathrm{~K}$ to agree with the higher $T_{\text {eff }}$ concluded from the He I lines.

Since Kurucz (2009, http://kurucz.harvard.edu/ atoms.html) has substantially extended his database, and the model atoms in our Tübingen Model-Atom Database (TMAD ${ }^{1}$ ) have been updated as well, we decided to calculate an improved, extended, state-of-the-art NLTE model-atmosphere grid. This grid is described in Sect. 2. The re-analysis of our UVES spectra (105-180 s, which in total cover one orbital period and which were also used by Müller et al. 2010) that were obtained in 2001 at the VLT is described in Sect. 3. We conclude in Sect. 4.

\section{Atomic data and model-atmosphere grid}

The model atmospheres used here were calculated with the Tübingen Model-Atmosphere Package (Werner et al. 2003, TMAP). The models are plane-parallel, in hydrostatic and radiative equilibrium. TMAP uses the occupation-probability formalism of Hummer \& Mihalas (1988) that was generalized to NLTE conditions by Hubeny et al. (1994). TMAP considers opacities of $\mathrm{H}+\mathrm{He}+\mathrm{C}+\mathrm{N}+\mathrm{O}+\mathrm{Mg}+\mathrm{Si}+\mathrm{P}+\mathrm{S}$ using classical model atoms, and $\mathrm{Ca}+\mathrm{Sc}+\mathrm{Ti}+\mathrm{V}+\mathrm{Cr}+\mathrm{Mn}+\mathrm{Fe}+\mathrm{Co}+\mathrm{Ni}$ uses a statistical approach (Rauch \& Deetjen 2003). All model atoms used in our calculations were updated to the most recent atomic data (Sect. 1), and 530 levels are treated in NLTE with 771 individual lines (from $\mathrm{H}-\mathrm{S}$ ) and 19957605 lines of $\mathrm{Ca}-\mathrm{Ni}$ from Kurucz'

${ }^{1}$ http://astro.uni-tuebingen.de/ TMAD/TMAD.html 
Table 1. Effective temperature and surface gravity of the primary of AA Dor, determined in previous and the present spectral analyses.

\begin{tabular}{|c|c|c|c|c|c|c|c|}
\hline $\begin{array}{l}T_{\text {eff }} \\
(\mathrm{K}) \\
\end{array}$ & $\begin{array}{c}\log g \\
\left(\mathrm{~cm} / \mathrm{s}^{2}\right) \\
\end{array}$ & $\begin{array}{c}\mathrm{He} \\
\text { (mass fraction) }\end{array}$ & $\begin{array}{c}v_{\mathrm{rot}}^{\mathrm{pri}} \\
\left(\mathrm{km} \mathrm{s}^{-1}\right) \\
\end{array}$ & $\begin{array}{l}M_{\text {pri }} \\
\left(M_{\odot}\right) \\
\end{array}$ & $\begin{array}{c}\left.M_{\mathrm{s}}\right) \\
\left(M_{\odot}\right) \\
\end{array}$ & Method & Reference \\
\hline 41000 & 5.4 & 0.28 & & & & $\mathrm{LTE}^{1}$ & Kudritzki (1976) \\
\hline 44200 & 5.2 & 0.28 & & & & NLTE $^{1}$ & Kudritzki (1976) \\
\hline 41700 & 5.9 & 0.8 & & & & $\mathrm{LTE}^{1}$ & Kudritzki (1976) \\
\hline 42000 & 5.7 & 0.8 & & & & NLTE $^{1}$ & Kudritzki (1976) \\
\hline $40000_{-2000}^{+3000}$ & $5.3 \pm 0.2$ & 0.012 & & 0.3 & 0.04 & NLTE $^{1}$ & Kudritzki et al. (1982) \\
\hline $42000 \pm 1000$ & $5.21 \pm 0.1$ & 0.0032 & 34.0 & 0.33 & 0.066 & NLTE $^{2}$ & Rauch (2000) \\
\hline $42000 \pm 1000$ & $5.30 \pm 0.1$ & 0.0032 & 35.0 & & & NLTE $^{3}$ & Fleig et al. (2008) \\
\hline $37800 \pm 500$ & $5.51 \pm 0.05$ & 0.005 & 30.0 & 0.51 & 0.085 & $\mathrm{LTE}^{4}$ & Müller et al. (2010) \\
\hline $42000 \pm 1000$ & $5.46 \pm 0.05$ & 0.0027 & 30.0 & 0.47 & 0.079 & NLTE $^{5}$ & this work \\
\hline
\end{tabular}

Notes. (1) $\mathrm{H}+\mathrm{He}$ models, two grids with fixed $N_{\mathrm{He}} / N_{\mathrm{H}}$ ratios only, 1st investigation of NLTE effects, no errors given. (2) $\mathrm{H}+\mathrm{He}+\mathrm{C}+\mathrm{N}+\mathrm{O}+\mathrm{Mg}+\mathrm{Si}+\mathrm{Fe}+\mathrm{Ni}, \mathrm{Fe}+\mathrm{Ni}$ data from Kurucz (1991), optical spectra, assumed bound rotation $\left(v_{\mathrm{rot}}^{\text {pri }}=45.7 \mathrm{~km} \mathrm{~s}^{-1}\right)$. (3) $\mathrm{H}+\mathrm{He}+\mathrm{C}+\mathrm{N}+\mathrm{O}+\mathrm{Mg}+\mathrm{Si}+\mathrm{P}+\mathrm{S}+\mathrm{Ca}+\mathrm{Sc}+\mathrm{Ti}+\mathrm{V}+\mathrm{Cr}+\mathrm{Mn}+\mathrm{Fe}+\mathrm{Co}+\mathrm{Ni}, \mathrm{Ca}-\mathrm{Ni}$ data from Kurucz (1991), optical and FUV spectra. ${ }^{(4)} \mathrm{H}+\mathrm{He}$ metal enhanced $\left(z=\right.$ ten times solar), optical spectra. ${ }^{(5)} \mathrm{H}+\mathrm{He}+\mathrm{C}+\mathrm{N}+\mathrm{O}+\mathrm{Mg}+\mathrm{Si}+\mathrm{P}+\mathrm{S}+\mathrm{Ca}+\mathrm{Sc}+\mathrm{Ti}+\mathrm{V}+\mathrm{Cr}+\mathrm{Mn}+\mathrm{Fe}+\mathrm{Co}+\mathrm{Ni}, \mathrm{Ca}-\mathrm{Ni}$ data from $\mathrm{Kurucz}$ (2009), optical spectra, $v_{\text {rot }}^{\text {pri }}$ from Müller et al. (2010).

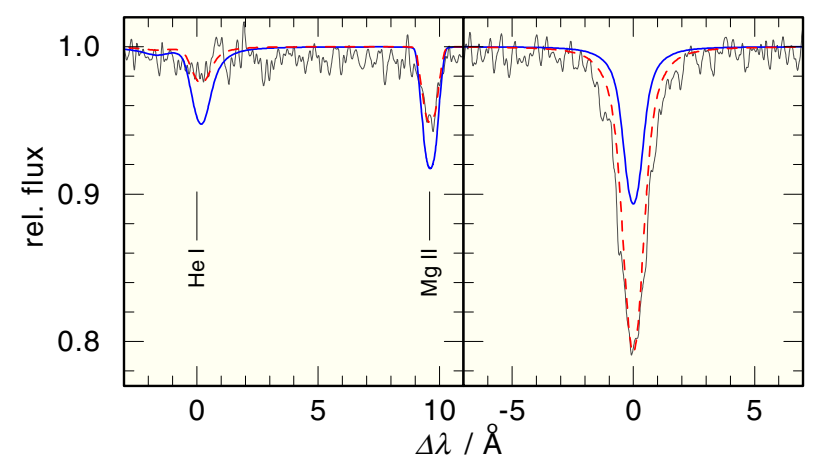

Fig. 1. Comparison of our synthetic spectra (full, blue line: $T_{\text {eff }}=37800 \mathrm{~K}, \log g=5.51$; dashed, red: $T_{\text {eff }}=42000 \mathrm{~K}, \log g=5.46$; $\mathrm{He}=0.0027$ by mass) around $\mathrm{He}$ I $\lambda 4471 \AA$ and $\mathrm{Mg}$ II $\lambda 4481 \AA$ (left) and He II $\lambda 4686 \AA$ (right) with the observation. The models are convolved with a rotational profile corresponding to $v_{\text {rot }}=30 \mathrm{~km} \mathrm{~s}^{-1}$. Models and observation are smoothed with a Gaussian $(0.1 \AA F W H M)$ for clarity.

line lists (Kurucz 2009) combined to 636 superlines. The element abundances are summarized in Table 2.

The model-atmosphere grid spans $T_{\text {eff }}=35000-49000 \mathrm{~K}$ $\left(\Delta T_{\text {eff }}=500 \mathrm{~K}\right)$ and $\log g=5.15-6.20(\Delta \log g=0.05)$. In total this makes 638 models. Spectral energy distributions (SEDs) were calculated using the most recent line broadening data, e.g. $\mathrm{H}_{\mathrm{I}}$ line-broadening has changed in TMAP since Fleig et al. (2008) presented their analysis of AA Dor. The reason is that Repolust et al. (2005) found an error in the Hi line-broadening tables (for high members of the spectral series only) by Lemke (1997) that were used before. These were substituted by a Holtsmark approximation. In addition, Tremblay \& Bergeron (2009) provide new, parameter-free Stark line-broadening tables for H I considering non-ideal effects. These replaced Lemke's data for the lowest ten members of the $\mathrm{H}_{\mathrm{I}}$ Lyman and Balmer series. In the parameter range of AADor, the new broadening tables have a significant impact on the line wings of higher Balmer-series members (narrower for $\mathrm{H} \epsilon$ and higher, Fig. 2). As a consequence, our analysis results in a higher $\log g$ (Sect. 3).

In the framework of the Virtual Observatory ${ }^{2}$ (VO), all these SEDs $\left(\lambda-F_{\lambda}\right)$ are available in VO compliant form via the

\footnotetext{
2 http://www.ivoa.net
}

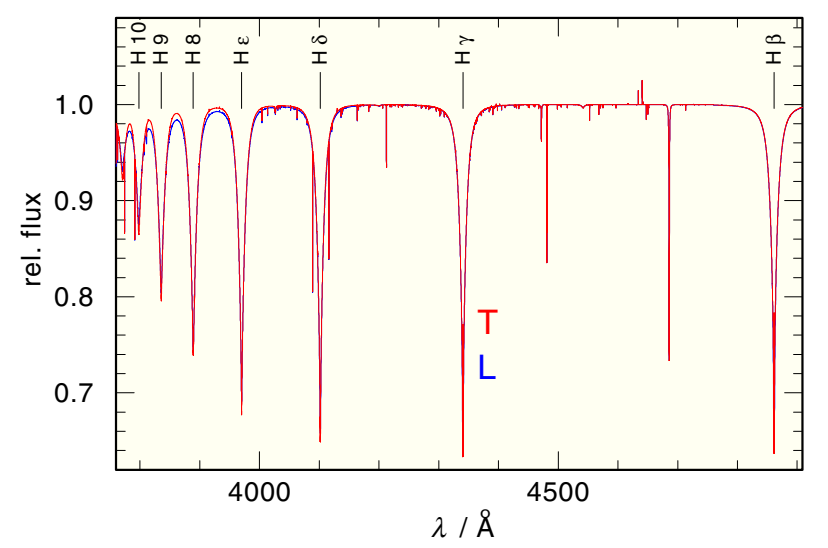

Fig. 2. Synthetic spectrum calculated from a $T_{\text {eff }}=42000 \mathrm{~K}$ and $\log g=5.45$ model with different Stark line-broadening tables (L, blue line: Lemke 1997, T, red: Tremblay \& Bergeron 2009, see text).

VO service TheoSSA ${ }^{3}$ provided by the German Astrophysical Virtual Observatory $\left(\mathrm{GAVO}^{4}\right)$.

\section{Analysis and results}

The light curve of AADor exhibits a reflection effect (e.g. Hilditch et al. 1996) that amounts to about $0.06 \mathrm{mag}$ in the optical. To analyze the pure primary spectrum, we selected only those four observations that were taken closest to the occultation of the secondary. These were co-added in order to improve the S/N. In Fig. 3, we show a $\chi^{2}$ fit to all single UVES spectra. Our $\chi^{2}$ fit excludes the inner line cores of $\mathrm{H} \beta$ and $\mathrm{H} \gamma$, as well as obviously bad data points (quality flags given by Müller et al. 2010). Both the occultation (at $\phi=0.5)$ and the transit $(\phi=0.0)$ of the secondary are clearly visible in the determination of $T_{\text {eff }}$ and $\log g$. Compared to a similar $\chi^{2}$ fit of Müller et al. (2010, their Fig. 3), we find the same $\log g=5.45$ but a significantly higher $T_{\text {eff }}=40500 \mathrm{~K}$ than for $T_{\text {eff }}=37800 \mathrm{~K}$.

For the analysis, we perform a detailed comparison in the classical way ( $\chi$-by-eye) and, for comparison in analogy to

\footnotetext{
${ }^{3}$ http://vo.ari.uni-heidelberg.de/ssatr-0.01/

TrSpectra.jsp?

${ }^{4}$ http://www.g-vo.org
} 


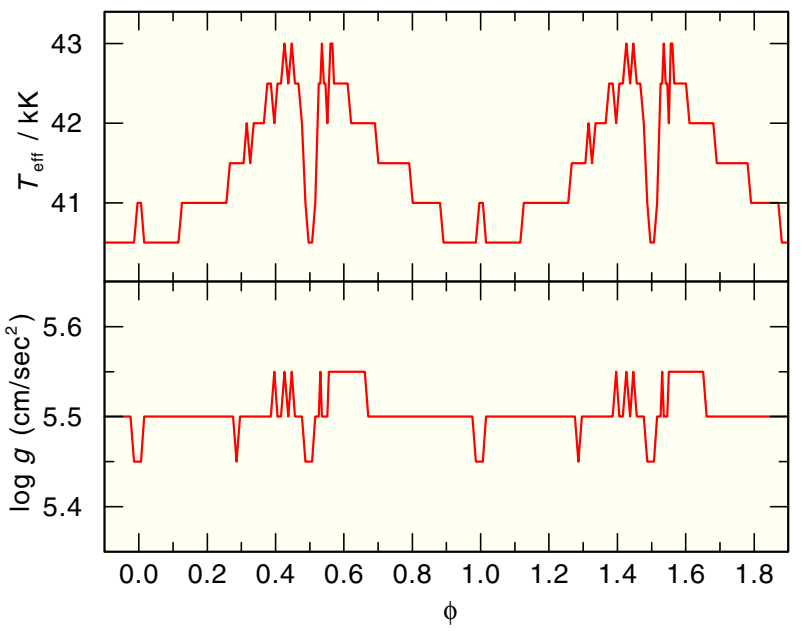

Fig. 3. Phase-dependent, best-fitting grid model determined by a $\chi^{2}$ fit. $\phi=0.0$ is the transit, $\phi=0.5$ is the occultation of the secondary. (The steps in $T_{\text {eff }}$ and $\log g$ represent the grid spacing.)

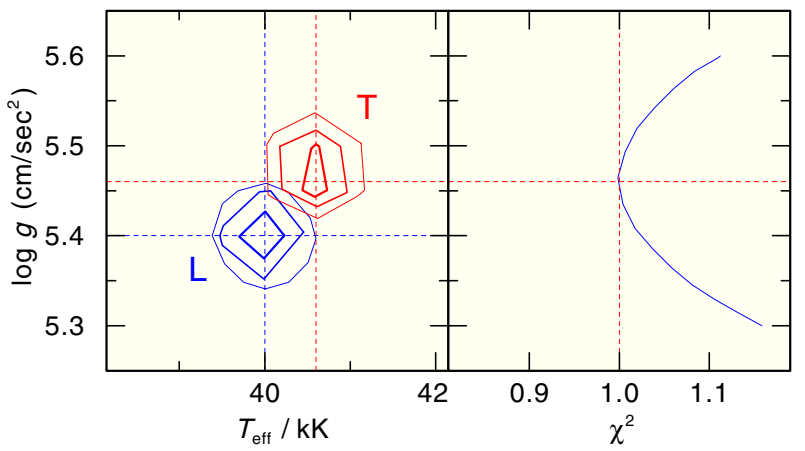

Fig. 4. Left: formal $1 \sigma, 2 \sigma$, and $3 \sigma$ contour lines of our $\chi^{2}$ fits in the $T_{\text {eff }}$ $-\log g$ plane. Right: reduced $\chi^{2}$ of our $T_{\text {eff }}=42000 \mathrm{~K}$ models depending on $\log g$.

Müller et al. (2010) with a $\chi^{2}$ fit, used the same wavelength limits (Table 3) and lines, $\mathrm{H} \beta-\mathrm{H} 11$ and He II $\lambda 4686 \AA$. Our $\chi^{2}$ fit yields $T_{\text {eff }}=40600 \pm 100 \mathrm{~K}$ and $\log g=5.46_{-0.02}^{+0.04}$ (T in Fig. 4). These errors are formal $1 \sigma$ errors, and $\sigma$ was calculated from the deviation of the $\chi_{\min }^{2}$ model from the observed spectrum used in the $\chi^{2}$ fit. Compared to a similar $\chi^{2}$ fit with SEDs that were calculated with the previously used Stark broadening tables of Lemke (1997, L in Fig. 4), there is a significant deviation of $\Delta T_{\text {eff }}=600 \mathrm{~K}$ and $\Delta \log g=0.06$.

A comparison of the best-fitting model from our $\chi^{2}$ fit and the best-fitting $\chi$-by-eye with the observations is shown in Fig. 5. It is obvious that the ionization equilibrium of $\mathrm{He}$ I/ $\mathrm{He}$ II is reproduced not at $T_{\text {eff }}=40600 \mathrm{~K}$ but at $T_{\text {eff }}=42000 \mathrm{~K}$. The theoretical line profiles of lower members of the Balmer series $(\mathrm{H} \beta-\mathrm{H} \delta)$ do not reproduce the observation perfectly. They fit slightly better at $T_{\text {eff }}=40600 \mathrm{~K}$. Thus, a small Balmer-line problem (Napiwotzki \& Rauch 1994; Werner 1996) due to additional metal opacities that are still not considered is apparently present. The inclusion of $\mathrm{He} \mathrm{I}_{\lambda} 4471 \AA$ (Table 3 ) in the $\chi^{2}$-fit procedure results in higher $T_{\text {eff }}=40700 \pm 300 \mathrm{~K}$. We finally adopt $T_{\text {eff }}=42000 \pm 1000 \mathrm{~K}$ (cf. Fleig et al. 2008) and $\log g=5.46 \pm 0.05$ because the previously evaluated ionization equilibria (Rauch 2000; Fleig et al. 2008) are an additional, crucial constraint. A $\chi^{2}$ fit at fixed $T_{\text {eff }}=42000 \mathrm{~K}$ (additional models were calculated with $\log g=5.30-5.60$ and $\Delta \log g=0.01$ ) also has its minimum at $\log g=5.46$ (Fig. 4).

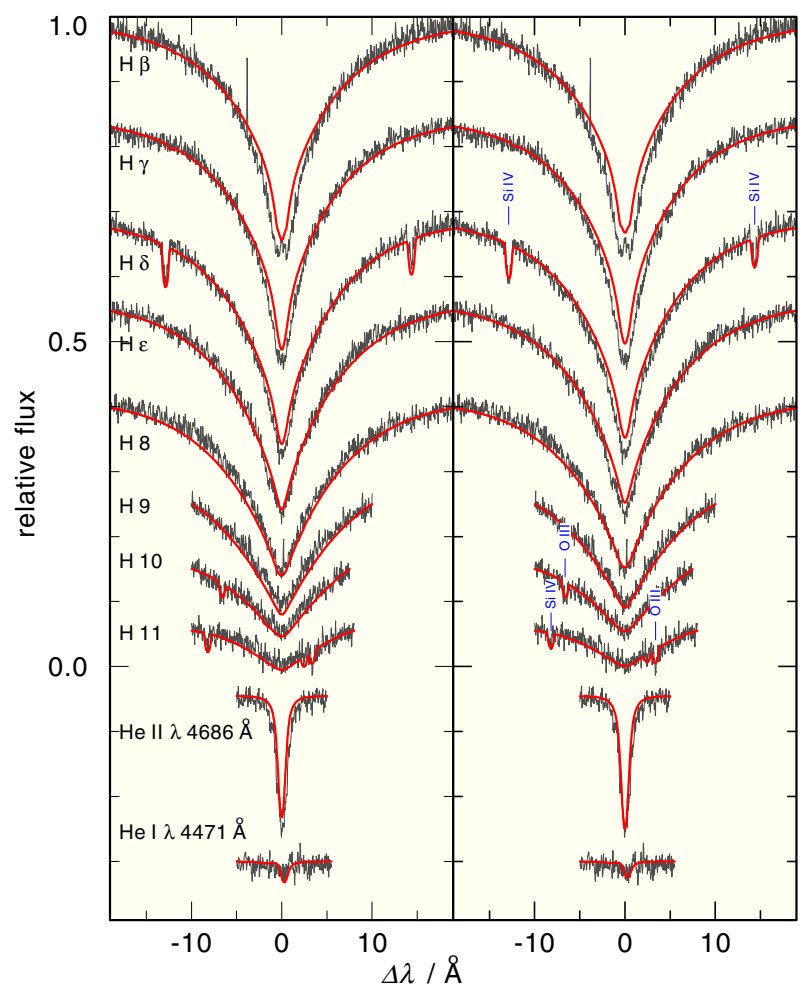

Fig. 5. Comparison of synthetic line profiles of $\mathrm{H}$ and $\mathrm{He}$ lines calculated from a $T_{\text {eff }}=40600 \mathrm{~K}$ and $\log g=5.46$ model (left) and a $T_{\text {eff }}=42000 \mathrm{~K}$ and $\log g=5.46$ model (right) with the observation.

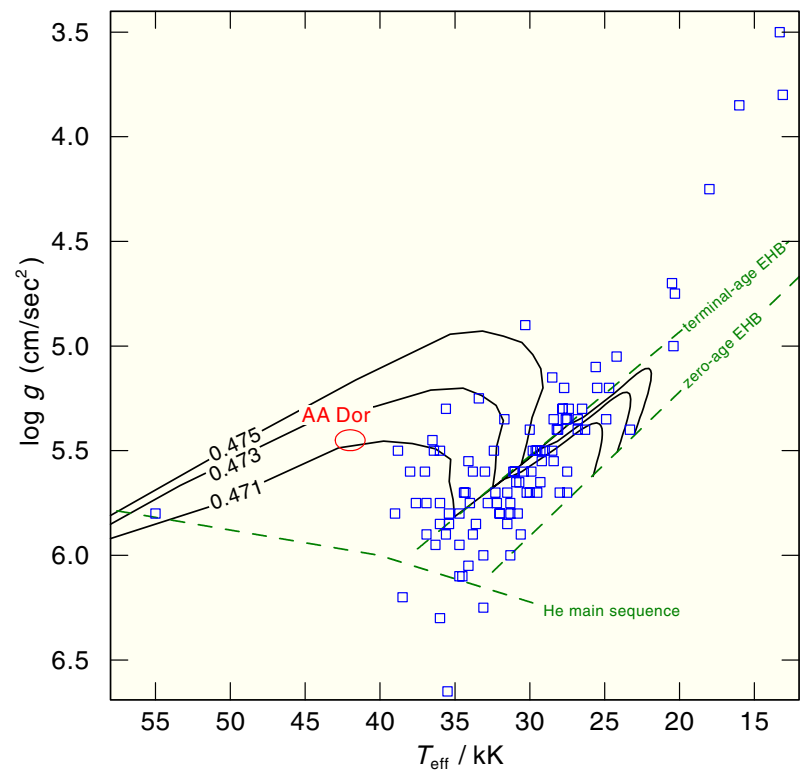

Fig. 6. Location of AA Dor in the $T_{\text {eff }}-\log g$ plane compared to sdBs and sdOBs from Edelmann (2003). Post-EHB tracks from Dorman et al. $\left(1998\right.$, labeled with the respective stellar masses in $M_{\odot}$ ) are also shown.

A mass of $M_{\text {pri }}=0.4714 \pm 0.0050 M_{\odot}$ is determined by comparing of $T_{\text {eff }}$ and $\log g$ with the evolutionary tracks of postEHB stars (Fig. 6). From the same evolutionary calculations, we interpolate the primary's luminosity. From our final model, we can determine the spectroscopic distance of AA Dor following Heber at al. (1984). We derive a distance of $d=352_{-23}^{+20} \mathrm{pc}$. The parameters of AA Dor are summarized in Tables 2 and 4. 
Table 4. Parameters of AA Dor compared with values of Hilditch et al. (2003).

\begin{tabular}{lcrl}
\hline \hline This work & & & Hilditch et al. (2003) \\
\cline { 1 - 2 }$T_{\text {eff }}^{\text {pri }} / \mathrm{kK}$ & $42.0 \pm 1$ & & \\
$\log \left(g_{\mathrm{pri}} / \frac{\mathrm{cm}}{\mathrm{s}^{2}}\right)$ & $5.46 \pm 0.05$ & & $5.45-5.51$ \\
$M_{\mathrm{pri}} / M_{\odot}$ & $0.4714 \pm 0.0050$ & & $0.33-0.47$ \\
$L_{\mathrm{pri}} / L_{\odot}$ & $120{ }_{-20}^{+15}$ & & \\
$M_{\mathrm{s}} / M_{\odot}$ & $0.0788_{-0.0075}^{+0.0075}$ & & $0.064-0.082$ \\
$d / \mathrm{pc}$ & $352{ }_{-23}^{+20}$ & & \\
\hline
\end{tabular}

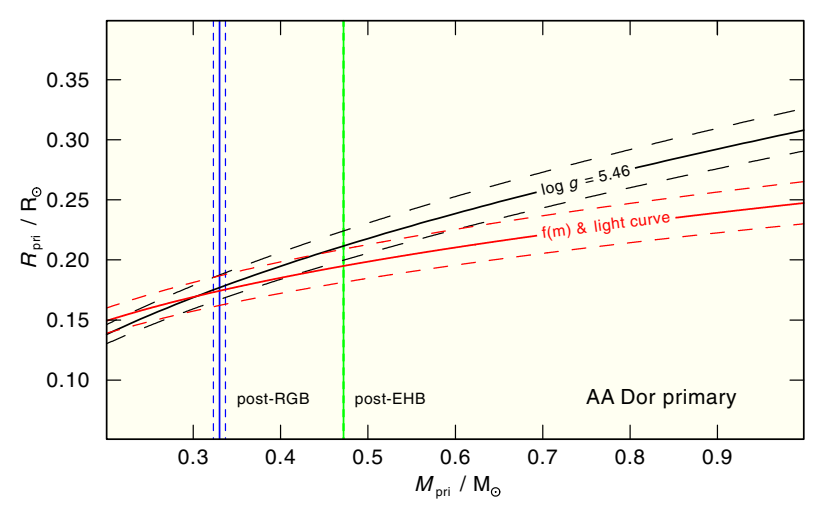

Fig. 7. Mass-radius relation for the primary of AA Dor. The dashed lines show the error ranges. The vertical lines show the primary mass, derived from comparison with post-RGB (Rauch 2000) and post-EHB (Fig. 6) evolutionary models.

\section{Conclusions}

The so-called $\log g$ problem in AADor is solved (Fig. 7) and our results (Table 4) are in good agreement with the photometric model of Hilditch et al. (2003).

Four influences were identified on the $\log g$ determination.

1) The major impact is the improvement in the Stark broadening tables, i.e. the difference between those of Lemke (1997) and of Tremblay \& Bergeron (2009). This results in a systematic deviation of $\Delta T_{\text {eff }}=600 \mathrm{~K}$ and $\Delta \log g=0.06$.

2) The reflection effect is now eliminated by using only observed spectra that were obtained during the occultation of the secondary (Sect. 3).

3) The improved atomic data makes the model-atmosphere more reliable thanks to a fuller consideration of the metalline blanketing. The temperature stratification of the stellar models, however, is only marginally affected.

4) The rotational velocity is lower than previously assumed (Müller et al. 2010). This only has little influence on the inner line core and is thus important for weak and narrow lines like He II $\lambda 4686 \AA$ (Fig. 1).

Since Vučković et al. (2008) identified spectral lines of the secondary in the UVES spectra and determined a lower limit $\left(K_{\mathrm{s}}>230 \mathrm{~km} \mathrm{~s}^{-1}\right)$ of its orbital velocity amplitude, both components' masses are known $\left(M_{\mathrm{pri}}=0.45 M_{\odot}, M_{\mathrm{s}}=0.076 M_{\odot}\right.$, Vučković et al. 2008), albeit with large error bars.
Müller et al. (2010) used the velocity amplitudes of both components $\left(K_{\text {pri }}=40.15 \pm 0.11 \mathrm{~km} \mathrm{~s}^{-1}, K_{\mathrm{s}}=240 \pm 20 \mathrm{~km} \mathrm{~s}^{-1}\right)$ to derive the masses $M_{\text {pri }}=0.51_{-0.108}^{+0.125} M_{\odot}$ and $M_{\mathrm{s}}=0.085_{-0.023}^{+0.031} M_{\odot}$. This rules out a post-RGB scenario because post-RGB masses are significantly lower. The solution from mass function $f(m)$ and light curve analysis, however, intersects with our result of $\log g=5.46 \pm 0.05$ (Fig. 7) even for the higher post-EHB mass (Fig. 6).

From our mass determination of $M_{\text {pri }}=0.4714 \pm 0.0050 M_{\odot}$, we calculated $\left(M_{\mathrm{pri}} K_{\mathrm{pri}}=M_{\mathrm{s}} K_{\mathrm{s}}\right)$ the secondary's mass of $M_{\mathrm{sec}}=$ $0.0725-0.0863 M_{\odot}$. Since the hydrogen-burning mass limit is about 0.075 $M_{\odot}$ (Chabrier \& Baraffe 1997; Chabrier et al. 2000), the secondary may either be a brown dwarf or a late M dwarf.

Acknowledgements. We thank the anonymous referee, the editor R. Napiwotzki, and K. Werner for comments and discussions that helped to improve the paper. The UVES spectra used in this analysis were obtained as part of an ESO Service Mode run, proposal 66.D-1800. This research made use of the SIMBAD Astronomical Database, operated at the CDS, Strasbourg, France. We thank the GAVO team for support. T.R. is supported by the German Aerospace Center (DLR) under grant 05 OR 0806.

\section{References}

Asplund, M., Grevesse, N., \& Sauval, A. J. 2009, ARA\&A, 47, 481 Chabrier, G., \& Baraffe, I. 1997, A\&A, 327, 1039

Chabrier, G., Baraffe, I., Allard, F., \& Hauschildt, P. 2000, ApJ, 542, 464 Dorman, B., Rood, R. T., \& O'Connell, R. W. 1998, ApJ, 419, 596

Edelmann, H. 2003, Ph.D. Thesis (Germany: University Erlangen-Nuremberg)

Fleig, J., Rauch, T., Werner, K., \& Kruk, J. W. 2008, A\&A, 492, 565

Heber, U., Hunger, K., Jonas, G., \& Kudritzki, R. P. 1984, A\&A, 130, 119

Hilditch, R. W., Harries, T. J., \& Hill, G. 1996, MNRAS, 279, 1380

Hilditch, R. W., Kilkenny, D., Lynas-Gray, A. E., \& Hill, G. 2003, MNRAS, 344, 644

Holweger, H. 1979, Les Élements et leurs Isotopes dans l'Univers, Université de Liège, Institue de Astrophysique, 117

Hubeny, I., Hummer, D. G., \& Lanz, T. 1994, A\&A, 282, 151

Hummer, D. G., \& Mihalas, D. 1988, ApJ, 331, 794

Kilkenny, D. 2011, MNRAS, 412, 487

Kudritzki, R. P. 1976, A\&A, 52, 11

Kudritzki, R. P., Simon, K. P., Lynas-Gray, A. E., Kilkenny, D., \& Hill, P. W. 1982, A\&A, 106, 254

Kurucz, R. L. 1991, in Stellar Atmospheres: Beyond Classical Models, ed. L. Crivellari, I. Hubeny, \& D. G. Hummer (Dordrecht: Kluwer, NATO ASI Series C), 341, 441

Kurucz, R. L. 2009, in Recent Directions in Astrophysical Quantitative Spectroscopy and Radiation Dynamics, ed. I. Hubeny, J. M. Stone, K. MacGregor, \& K. Werner, AIP Conf. Ser. 1171, 43

Lemke, M. 1997, A\&AS, 122, 285

Müller, S., Geier, S., \& Heber, U. 2010, Ap\&SS, 329, 101

Napiwotzki, R., \& Rauch, T. 1994, A\&A, 285, 603

Rauch, T. 2000, A\&A, 356, 665

Rauch, T. 2004, Ap\&SS, 291, 275

Rauch, T., \& Deetjen, J. L. 2003, in Stellar Atmosphere Modeling, ed. I. Hubeny, D. Mihalas, \& K. Werner, ASP Conf. Ser., 288, 103

Rauch, T., Dreizler, S., \& Wolff, B. 1998, A\&A, 338, 651

Repolust, T., Puls, J., Hanson, M. M., Kudritzki, R.-P., \& Mokiem, M. R. 2005, A\&A, 440, 261

Tremblay, P.-E., \& Bergeron, P. 2009, ApJ, 696, 1755

Vučković, M., Østensen, R., Bloemen, S., Decoster, I., \& Aerts, C. 2008, in Hot Subdwarf Stars and Related Objects, ed. U. Heber, C. S. Jeffery, \& R. Napiwotzki, ASP Conf. Ser., 392, 199

Werner, K. 1996, ApJ, 457, L 39

Werner, K., Dreizler, S., Deetjen, J. L., et al. 2003, in Stellar Atmosphere Modeling, ed. I. Hubeny, D. Mihalas, \& K. Werner, ASP Conf. Ser., 288, 31 
S. Klepp and T. Rauch: Non-LTE spectral analysis of the sdOB primary of AA Dor

Table 2. Element abundances in our model-atmosphere grid.

\begin{tabular}{crlrl}
\hline \hline $\begin{array}{c}\text { Element } \\
\mathrm{X}\end{array}$ & $\begin{array}{r}\text { Mass } \\
\text { fraction }\end{array}$ & $\begin{array}{l}\text { Number } \\
\text { fraction }\end{array}$ & {$\left[\epsilon_{\mathrm{X}}\right]^{*}$} & {$[\mathrm{X}]^{* *}$} \\
\hline $\mathrm{H}$ & $9.939 \mathrm{E}-1$ & $9.992 \mathrm{E}-1$ & 12.144 & +0.130 \\
$\mathrm{He}$ & $2.686 \mathrm{E}-3$ & $6.801 \mathrm{E}-4$ & 8.977 & -1.967 \\
$\mathrm{C}$ & $1.777 \mathrm{E}-5$ & $1.499 \mathrm{E}-6$ & 6.320 & -2.124 \\
$\mathrm{~N}$ & $4.145 \mathrm{E}-5$ & $2.998 \mathrm{E}-6$ & 6.621 & -1.223 \\
$\mathrm{O}$ & $1.009 \mathrm{E}-3$ & $6.393 \mathrm{E}-5$ & 7.950 & -0.754 \\
$\mathrm{Mg}$ & $4.078 \mathrm{E}-4$ & $1.700 \mathrm{E}-5$ & 7.375 & -0.240 \\
$\mathrm{Si}$ & $3.049 \mathrm{E}-4$ & $1.100 \mathrm{E}-5$ & 7.186 & -0.339 \\
$\mathrm{P}$ & $5.197 \mathrm{E}-6$ & $1.700 \mathrm{E}-7$ & 5.375 & -0.050 \\
$\mathrm{~S}$ & $3.241 \mathrm{E}-6$ & $1.024 \mathrm{E}-7$ & 5.155 & -1.980 \\
$\mathrm{Ca}$ & $5.994 \mathrm{E}-5$ & $1.515 \mathrm{E}-6$ & 6.325 & -0.030 \\
$\mathrm{Sc}$ & $3.694 \mathrm{E}-8$ & $8.327 \mathrm{E}-0$ & 3.065 & -0.100 \\
$\mathrm{Ti}$ & $2.784 \mathrm{E}-6$ & $5.894 \mathrm{E}-8$ & 4.915 & -0.050 \\
$\mathrm{~V}$ & $3.731 \mathrm{E}-7$ & $7.421 \mathrm{E}-9$ & 4.015 & +0.070 \\
$\mathrm{Cr}$ & $1.663 \mathrm{E}-5$ & $3.240 \mathrm{E}-7$ & 5.655 & +0.001 \\
$\mathrm{Mn}$ & $9.877 \mathrm{E}-6$ & $1.828 \mathrm{E}-7$ & 5.405 & -0.040 \\
$\mathrm{Fe}$ & $1.153 \mathrm{E}-3$ & $2.091 \mathrm{E}-5$ & 7.465 & -0.050 \\
$\mathrm{Co}$ & $3.591 \mathrm{E}-6$ & $6.174 \mathrm{E}-8$ & 4.935 & -0.069 \\
$\mathrm{Ni}$ & $3.482 \mathrm{E}-4$ & $6.009 \mathrm{E}-6$ & 6.923 & +0.689 \\
\hline
\end{tabular}

Notes. (*) $\log \left(\epsilon_{\mathrm{i}} / \epsilon_{\odot}\right), \log \sum_{\mathrm{i}} \mu_{\mathrm{i}} \epsilon_{\mathrm{i}}=12.15$, (cf. Holweger 1979). $\left.{ }^{(* *}\right) \log$ [abundance/solar abundance] (solar values from Asplund et al. 2009).
Table 3. Lines and wavelength intervals used for our $\chi^{2}$ fits.

\begin{tabular}{lccc}
\hline \hline Line & $\begin{array}{c}\Delta \lambda \\
(\AA)\end{array}$ & Line & $\begin{array}{c}\Delta \lambda \\
(\AA)\end{array}$ \\
\hline $\mathrm{H} \beta$ & {$[-50,+50]$} & He I $\lambda 4471 \AA$ & {$[-5,+5]$} \\
$\mathrm{H} \gamma$ & {$[-40,+40]$} & He II $\lambda 4686 \AA$ & {$[-5,+5]$} \\
$\mathrm{H} \delta$ & {$[-30,+30]$} & & \\
$\mathrm{H} \epsilon$ & {$[-20,+20]$} & & \\
$\mathrm{H} 8$ & {$[-20,+20]$} & & \\
$\mathrm{H} 9$ & {$[-10,+10]$} & & \\
H 10 & {$[-10,+7]$} & & \\
H 11 & {$[-10,+8]$} & & \\
\hline
\end{tabular}

